\title{
Green function approach to the theory of superconductivity in the $t-J$ model
}

\author{
N.M.Plakida \\ Joint Institute for Nuclear Research, 141980 Dubna, Russia \\ Received January 28, 1998 \\ The theory of superconducting pairing due to the exchange and kinemati- \\ cal interactions in the $t-J$ model in a paramagnetic state is developed. \\ The Dyson equation for the matrix Green functions in terms of the Hubbard \\ operators is obtained in the noncrossing approximation. The linearized self- \\ consistent system of Eliashberg equations is proposed to study the tem- \\ perature and doping dependence of the quasi-particle hole spectrum in \\ the normal state and to calculate the temperature of the superconducting \\ phase transition and the symmetry of the gap function.
}

Key words: superconductivity, quasi-particle, Dyson equation, Green functions, Hubbard operators

PACS: 74.20.-z, 74.20.Mn, 74.72.-h

\section{Introduction}

In 1960 D.N. Zubarev [1] proposed a theory of superconductivity for an electron-phonon system based on the equation of motion method for two-time Green functions [2]. The paper was published simultaneously with the famous papers by G.M. Eliashberg [3] where the temperature diagram technique was used to obtain the Gorkov type equations for an electron-phonon system. However, Zubarev's formulation did not draw proper attention of the following investigators, while the Eliashberg theory was cited frequently in many papers and his formulation became known as the Eliashberg (or Migdal-Eliashberg) theory of superconductivity for an electron-phonon system. The real advantage of the Eliashberg formulation is that it permits one to consider a strong coupling limit by using the skeleton diagram technique. In the Zubarev formulation based on a successive differentiation of the Green functions over the same time one cannot employ the skeleton diagram technique. However, in the two-time differentiation method for the Green functions this problem can be easily overcome and the Eliashberg type equations, as it has been shown in [4], can be formulated in a very simple and transparent way for a general model of an electron boson-field interaction. 
In the present paper the theory of superconductivity is formulated for the $t-J$ model by applying an equation of motion method for the Green functions in terms of the Hubbard operators. It should be pointed out that superconducting pairing due to the kinematical interaction in the Hubbard model in the limit of strong electron correlations $(U \rightarrow \infty)$ was first obtained by Zaitsev and Ivanov [5]. However, they considered only the mean field approximation which results in the $s$-wave pairing irrelevant for strongly correlated systems (for a discussion see [6]). Later on the theory in the mean field approximation was considered for the $t-J$ model within the Green function approach in $[6,7]$ where $d$-wave spin fluctuation superconducting pairing was obtained due to exchange interaction $J$.

In the present formulation we have calculated a matrix self-energy operator in the noncrossing approximation for kinematical and exchange interactions which neglects vertex corrections, as in the Migdal-Eliashberg theory. The self-energy operator allowing for the finite life-time effects for electrons plays an essential role both in the renormalization of the quasiparticle spectrum and in superconducting pairing. It was clearly demonstrated in [8] where the $t-J$ model in the polaron representation was considered. A self-consistent numerical solution of the Eliashberg equations has proved a strong renormalization of the quasiparticle hole spectrum due to spin-fluctuations and $d$-wave pairing at a finite concentration of doped holes. However, the two-sublattice representation used in [8] can be rigorously proved only for a small doping. At a moderate doping one has to consider the paramagnetic (spin-rotationally invariant) state in the $t-J$ model. In that case the Hubbard operator technique is useful. However, due to unconventional commutation relations the Hubbard operators cannot be treated within the standard diagram technique (see, e.g., [9]). To overcome this problem one can employ different types of the slave-boson (-fermion) technique to use the standard fermionboson diagram technique. However, due to crude treatment of constraints which should be observed in the slave-field techniques the results of that calculation appear to be quite unreliable.

The two-time Green function approach in terms of the Hubbard operators was also used to consider superconducting pairing in the $t-J$ model with an electronphonon interaction in [10] and to discuss the electron and hole spectra in the normal state in [11]. A general discussion concerning the $t-J$ model and its applications in the description of physical properties of the systems with strong electron correlations, as copper-oxide superconductors, can be found, e.g., in [12-14].

The paper is organized as follows. In the next section the $t-J$ model in terms of the Hubbard operators is introduced. In section 3 the Dyson equation for the matrix Green function is obtained by the projection technique in the equation of motion method. In section 4 a self-consistent system of Eliashberg equations in the noncrossing (self-consistent) approximation is formulated and the linearized gap equation for the calculation of $T_{\mathrm{c}}$ is presented. Conclusions are given in section 5 . 


\section{The $t-J$ model}

The simplest model allowing for the electron correlations in copper oxides is the one-band Hubbard model [15]:

$$
H=-t \sum_{i j \sigma} a_{i \sigma}^{+} a_{j \sigma}+U \sum_{i} n_{i \uparrow} n_{i \downarrow}
$$

where $t$ is an effective transfer integral and $U$ is the Coulomb one-site energy. In the strong coupling limit, $U \gg t$, we can reduce the Hubbard model (2.1) (or a more realistic for copper oxides $p-d$ model [16]) to the $t-J$ model [17]:

$$
H_{t-J}=-\sum_{i \neq j, \sigma} t_{i j} \tilde{c}_{i \sigma}^{+} \tilde{c}_{j \sigma}+J \sum_{\langle i j\rangle}\left(\mathbf{S}_{i} \mathbf{S}_{j}-\frac{1}{4} n_{i} n_{j}\right),
$$

where the first term describes electron hopping with the energy $t_{i j}$ for the nearest neighbours, $t_{i j}=t$, for the second neighbours, $t_{i j}=t^{\prime}$, etc., on a two dimensional square lattice. The electron operators $\tilde{c}_{i \sigma}^{+}=c_{i \sigma}^{+}\left(1-n_{i-\sigma}\right)$ act in the space without double occupancy and $n_{i}=n_{i \uparrow}+n_{i \downarrow}$ is the number operator for electrons. The second term describes the spin-1/2 Heisenberg antiferromagnet (AFM) with the exchange energy $J$ for the nearest neighbours which is equal to $J=4 t^{2} / U$ for the Hubbard model (2.1) or can be considered as an independent parameter in the case of the $p-d$ model. In the model (2.2) two main features of a doped hole motion in copper-oxides are properly taken into account: a constraint on no double occupancy for the holes on lattice sites due to strong electron correlations and an interaction of holes with AFM spin fluctuations which result in strong renormalization of the quasiparticle spectrum (for a review, see [14]).

To take into account on a rigorous basis the exclusion of doubly occupied states in electronic hopping, we employ the Hubbard operator (HO) technique. The HOs are defined as

$$
X_{i}^{\alpha \beta}=|i, \alpha\rangle\langle i, \beta|
$$

for the three possible states at the lattice site $i$

$$
|i, \alpha\rangle=|i, 0\rangle, \quad|i, \sigma\rangle,
$$

for an empty site and for a singly occupied site by an electron with spin $\sigma / 2$ $(\sigma= \pm 1)$. In the $t-J$ model only singly occupied sites are retained and the completeness relation for the HOs reads:

$$
X_{i}^{00}+\sum_{\sigma} X_{i}^{\sigma \sigma}=1
$$

The spin and density operators in equation (2.2) are expressed by HOs as

$$
S_{i}^{\sigma}=X_{i}^{\sigma \bar{\sigma}}, \quad S_{i}^{z}=\frac{1}{2} \sum_{\sigma} \sigma X_{i}^{\sigma \sigma}, \quad n_{i}=\sum_{\sigma} X_{i}^{\sigma \sigma}
$$


where $\bar{\sigma}=-\sigma$. The HOs obey the following multiplication rules

$$
X_{i}^{\alpha \beta} X_{i}^{\gamma \delta}=\delta_{\beta \gamma} X_{i}^{\alpha \delta}
$$

and commutation relations

$$
\left[X_{i}^{\alpha \beta}, X_{j}^{\gamma \delta}\right]_{ \pm}=\delta_{i j}\left(\delta_{\beta \gamma} X_{i}^{\alpha \delta} \pm \delta_{\delta \alpha} X_{i}^{\gamma \beta}\right) .
$$

In equation (2.8) the upper sign stands for the case when both HOs are Fermi-like ones (as, e.g., $X_{i}^{0 \sigma}$ ). The spin and density operators (2.6) are Bose-like and for them the lower sign in equation (2.8) should be taken.

The Hamiltonian of the $t-J$ model (2.2) in terms of HOs reads

$$
H_{t-J}=-\sum_{i \neq j, \sigma} t_{i j} X_{i}^{\sigma 0} X_{j}^{0 \sigma}-\mu \sum_{i \sigma} X_{i}^{\sigma \sigma}+\frac{1}{2} \sum_{i \neq j, \sigma} J_{i j}\left(X_{i}^{\sigma \bar{\sigma}} X_{j}^{\bar{\sigma} \sigma}-X_{i}^{\sigma \sigma} X_{j}^{\bar{\sigma} \bar{\sigma}}\right),
$$

where the exchange interaction is written in a more general form with the exchange energy $J_{i j}$ for the lattice sites $(i, j)$.

The unconventional commutation relations (2.8) hamper the treatment of the model within the standard diagrammatic technique. To overcome this problem we will use the equation of motion method for the two-time Green functions [2] in terms of the HOs (2.3) which rigorously preserve the constraint of no-double occupancy.

\section{Dyson equation for the matrix Green function}

To discuss superconducting pairing within the model (2.9) we consider the matrix Green function (GF)

$$
\hat{G}_{i j, \sigma}\left(t-t^{\prime}\right)=\left\langle\left\langle\Psi_{i \sigma}(t) \mid \Psi_{j \sigma}^{+}\left(t^{\prime}\right)\right\rangle\right\rangle
$$

in terms of the Nambu operators:

$$
\Psi_{i \sigma}=\left(\begin{array}{c}
X_{i}^{0 \sigma} \\
X_{i}^{\bar{\sigma} 0}
\end{array}\right), \quad \Psi_{i \sigma}^{+}=\left(X_{i}^{\sigma 0} X_{i}^{0 \bar{\sigma}}\right) .
$$

Here Zubarev's notations for the anticommutator Green function (3.1) are used [2].

By differentiating the GF (3.1) over the time $t$ we get the following equation for the Fourier component:

$$
\omega \hat{G}_{i j \sigma}(\omega)=\delta_{i j} \hat{Q}_{\sigma}+\left\langle\left\langle\hat{Z}_{i \sigma} \mid \Psi_{j \sigma}\right\rangle\right\rangle_{\omega}
$$

where $\hat{Z}_{i \sigma}=\left[\Psi_{i \sigma}, H\right]$ and

$$
\hat{Q}_{\sigma}=\left(\begin{array}{cc}
Q_{\sigma} & 0 \\
0 & Q_{\bar{\sigma}}
\end{array}\right)
$$

with the matrix elements $Q_{\sigma}=\left\langle X_{i}^{00}+X_{i}^{\sigma \sigma}\right\rangle$. Here and in what follows we consider a spin-singlet state for which the correlation functions do not depend on the spin $\sigma$. 
In that case, by using equation $(2.5)$ we get $Q_{\sigma}=Q=1-n / 2$ where the average number of electrons is given by the equation

$$
n=\left\langle n_{i}\right\rangle=\sum_{\sigma}\left\langle X_{i}^{\sigma \sigma}\right\rangle .
$$

Now, we project the many-particle GF in (3.3) on the one-hole one by introducing an irreducible $(i r r)$ part of the $\hat{Z}_{i \sigma}$ operator

$$
\left\langle\left\langle\hat{Z}_{i \sigma} \mid \Psi_{j \sigma}^{+}\right\rangle\right\rangle=\sum_{l} \hat{E}_{i l \sigma}\left\langle\left\langle\Psi_{l \sigma} \mid \Psi_{j \sigma}^{+}\right\rangle\right\rangle+\left\langle\left\langle\hat{Z}_{i \sigma}^{(\mathrm{irr})} \mid \Psi_{j \sigma}^{+}\right\rangle\right\rangle
$$

The projection is defined by the condition

$$
\left\langle\left\{\hat{Z}_{i \sigma}^{(\mathrm{irr})}, \Psi_{j \sigma}^{+}\right\}\right\rangle=0,
$$

that results in the equation for the frequency matrix

$$
\hat{E}_{i j \sigma}=\left\langle\left\{\left[\Psi_{i \sigma}, H\right], \Psi_{j \sigma}^{+}\right\}\right\rangle \hat{Q}_{\sigma}^{-1} .
$$

Here $\{A, B\}$ and $[A, B]$ are the anticommutator and the commutator for the $A, B$ operators, respectively. To calculate the matrix (3.8) we use the equation of motion for the HOs as, e.g.,

$$
\left(\mathrm{i} \frac{\mathrm{d}}{\mathrm{d} t}+\mu\right) X_{i}^{0 \sigma}=\sum_{l} t_{i l} B_{i \sigma \sigma^{\prime}} X_{l}^{0 \sigma^{\prime}}+\sum_{l} J_{i l}\left(B_{l \sigma \sigma^{\prime}}-\delta_{\sigma \sigma^{\prime}}\right) X_{i}^{0 \sigma^{\prime}},
$$

where we introduced the operator

$$
B_{i \sigma \sigma^{\prime}}=\left(X_{i}^{00}+X_{i}^{\sigma \sigma}\right) \delta_{\sigma^{\prime} \sigma}+X_{i}^{\bar{\sigma} \sigma} \delta_{\sigma^{\prime} \bar{\sigma}}
$$

The Bose-like operator (3.10) describes electron scattering on spin and charge fluctuations caused by the nonfermionic commutation relations for the HOs (the first term in (3.9) - the so-called kinematical interaction) and by the exchange spinspin interaction (the second term in (3.9)). It can be demonstrated explicitly by using the completeness relation (2.5) that results in the following representation:

$$
\begin{aligned}
X_{i}^{00}+X_{i}^{\sigma \sigma}+X_{i}^{\bar{\sigma} \sigma} & =1-\frac{1}{2} \sum_{\sigma} X_{i}^{\sigma \sigma}+\frac{1}{2}\left(X_{i}^{\sigma \sigma}-X_{i}^{\bar{\sigma} \bar{\sigma}}\right)+X_{i}^{\bar{\sigma} \sigma} \\
& =1-\frac{1}{2} n_{i}+\sigma S_{i}^{z}+S_{i}^{\bar{\sigma}} .
\end{aligned}
$$

By performing commutations in (3.8), we get for the normal and the anomalous parts of the frequency matrix:

$$
\begin{aligned}
E_{i j \sigma}^{11}= & \delta_{i j} \sum_{l}\left\{t_{i l}\left\langle X_{i}^{\sigma 0} X_{l}^{0 \sigma}\right\rangle / Q_{\sigma}+J_{i l}\left(Q_{\sigma}-1+\chi_{i l}^{\mathrm{cs}} / Q_{\sigma}\right)\right\} \\
& -t_{i j}\left(Q_{\sigma}+\chi_{i j}^{\mathrm{cs}} / Q_{\sigma}\right)-J_{i j}\left\langle X_{j}^{\sigma 0} X_{i}^{0 \sigma}\right\rangle / Q_{\sigma} \\
E_{i j \sigma}^{12}= & \delta_{i j} \sum_{l} t_{i l}\left\langle X_{i}^{0 \bar{\sigma}} X_{l}^{0 \sigma}+X_{l}^{0 \bar{\sigma}} X_{i}^{0 \sigma}\right\rangle / Q_{\sigma} \\
& -J_{i j}\left\langle X_{i}^{0 \bar{\sigma}} X_{j}^{0 \sigma}+X_{j}^{0 \bar{\sigma}} X_{i}^{0 \sigma}\right\rangle / Q_{\sigma}
\end{aligned}
$$


Here we introduce the charge- and spin-fluctuation correlation functions

$$
\chi_{i j}^{\mathrm{cs}}=\frac{1}{4}\left\langle\delta n_{i} \delta n_{j}\right\rangle+\left\langle\mathbf{S}_{\mathbf{i}} \mathbf{S}_{\mathbf{j}}\right\rangle,
$$

with $\delta n_{i}=n_{i}-\left\langle n_{i}\right\rangle$.

Now we introduce the zero-order GF in the generalized mean-field approximation by neglecting the finite lifetime effects described by the operator $\hat{Z}_{i \sigma}^{\text {(irr) }}$ in equation (3.6)

$$
\hat{G}_{i j \sigma}^{0}(\omega)=\left\{\omega \hat{\tau}_{0} \delta_{i j}-\hat{E}_{i j \sigma}\right\}^{-1} \hat{Q}_{\sigma}
$$

where $\hat{\tau}_{0}$ is the unity matrix. By writing the equation of motion for the irreducible part of the GF in (3.6) with respect to the second time $t^{\prime}$ for the right-hand side operator $\Psi_{j \sigma}^{+}\left(t^{\prime}\right)$ and performing the same projection procedure as in (3.6), we get

$$
\left\langle\left\langle\hat{Z}_{i \sigma}^{(\mathrm{irr})} \mid \Psi_{j \sigma}^{+}\right\rangle\right\rangle_{\omega}=\sum_{l}\left\langle\left\langle\hat{Z}_{i \sigma}^{(\mathrm{irr})} \mid\left(Z_{l \sigma}^{(\mathrm{irr})}\right)^{+}\right\rangle\right\rangle_{\omega} \hat{Q}_{\sigma}^{-1} \hat{G}_{l j \sigma}^{0}(\omega) .
$$

By using (3.3), (3.6) and (3.15), we can obtain the Dyson equation for the GF (3.1) in the form:

$$
\hat{G}_{i j \sigma}(\omega)=\hat{G}_{i j \sigma}^{0}(\omega)+\sum_{k l} \hat{G}_{i k \sigma}^{0}(\omega) \hat{\Sigma}_{k l \sigma}(\omega) \hat{G}_{l j \sigma}(\omega)
$$

where the self-energy operator $\hat{\Sigma}_{k l \sigma}(\omega)$ is defined by the equation

$$
\hat{T}_{i j \sigma}(\omega)=\hat{\Sigma}_{i j \sigma}(\omega)+\sum_{k l} \hat{\Sigma}_{i k \sigma}(\omega) \hat{G}_{k l \sigma}^{0}(\omega) \hat{T}_{l j \sigma}(\omega)
$$

Here the scattering matrix is given by the equation

$$
\hat{T}_{i j \sigma}(\omega)=\hat{Q}_{\sigma}^{-1}\left\langle\left\langle\hat{Z}_{i \sigma}^{(\mathrm{irr})} \mid \hat{Z}_{j \sigma}^{(\mathrm{irr})^{+}}\right\rangle\right\rangle_{\omega}{\hat{Q_{\sigma}}}^{-1}
$$

From equation (3.17) it follows that the self-energy operator is given by the irreducible part of the scattering matrix (3.18) which has no single zero-order GF (3.14) lines:

$$
\hat{\Sigma}_{i j \sigma}(\omega)={\hat{Q_{\sigma}}}^{-1}\left\langle\left\langle\hat{Z}_{i \sigma}^{(\mathrm{irr})} \mid \hat{Z}_{j \sigma}^{(\mathrm{irr})^{+}}\right\rangle\right\rangle_{\omega}^{(\mathrm{irr})}{\hat{Q_{\sigma}}}^{-1} .
$$

Equations (3.14), (3.16) and (3.19) give an exact representation for the onehole GF (3.1). To calculate it, however, one has to apply some approximations for the many-particle GF in the self-energy matrix (3.19) which describes inelastic scattering of electrons on spin and charge fluctuations.

\section{Self-consistent Eliashberg equations}

To solve the Dyson equation (3.16) we introduce the k-representation for the GF

$$
G_{\sigma}^{\alpha \beta}(k, \omega)=\sum_{j} G_{o j \sigma}^{\alpha \beta}(\omega) \mathrm{e}^{-\mathrm{i} \mathbf{k j}}
$$


For the zero-order GF (3.14) we get:

$$
\hat{G}_{\sigma}^{(0)}(k, \omega)^{-1}=\left\{\omega \hat{\tau}_{0}-\left(E_{k}^{\sigma}-\tilde{\mu}\right) \hat{\tau}_{3}-\Delta_{k}^{\sigma} \hat{\tau}_{1}\right\} \hat{Q}_{\sigma}^{-1},
$$

where $\hat{\tau}_{0}, \hat{\tau}_{1}, \hat{\tau}_{3}$ are the Pauli matrix. The energy of the quasiparticles $E_{k}^{\sigma}$, the renormalized chemical potential $\tilde{\mu}=\mu-\delta \mu$ and the gap function $\Delta_{k}^{\sigma}$ in the MFA, equations (3.11), (3.12), are given by

$$
E_{k}^{\sigma}=-\epsilon(k) Q_{\sigma}-\epsilon_{s}(k) / Q_{\sigma}-\frac{4 J}{N} \sum_{q} \gamma(k-q) N_{q \sigma}
$$

where

$$
\begin{aligned}
& \epsilon(k)=t(k)=4 t \gamma(k)+4 t^{\prime} \gamma^{\prime}(k), \quad \epsilon_{s}(k)=4 t \gamma(k) \chi_{1 s}+4 t^{\prime} \gamma^{\prime}(k) \chi_{2 s}, \\
& \gamma(k)=(1 / 2)\left(\cos a_{x} q_{x}+\cos a_{y} q_{y}\right), \quad \gamma^{\prime}(k)=\cos a_{x} q_{x} \cos a_{y} q_{y},
\end{aligned}
$$

with

$$
\begin{gathered}
\delta \mu=\frac{1}{N} \sum_{q} \epsilon(q) N_{q \sigma}-4 J\left(n / 2-\chi_{1 s} / Q_{\sigma}\right), \\
\Delta_{k}^{\sigma}=\frac{2}{N Q_{\sigma}} \sum_{q} J(k-q)\left\langle X_{-q}^{0 \bar{\sigma}} X_{q}^{0 \sigma}\right\rangle .
\end{gathered}
$$

The average number of electrons (3.5) in the $\mathbf{k}$-representation is written in the form:

$$
n=\frac{1}{N} \sum_{k, \sigma}\left\langle X_{k}^{\sigma 0} X_{k}^{0 \sigma}\right\rangle=\frac{1}{N} \sum_{k, \sigma} Q_{\sigma} N_{k \sigma},
$$

which defines the function $N_{q \sigma}$ in equations (4.3), (4.4). In the calculation of the normal part of the frequency matrix (4.3) we neglected the charge fluctuation (the first term in equation (3.13)) and introduced the spin correlation functions for the nearest $\left(\chi_{1 \mathrm{~s}}\right)$ and the next-nearest $\left(\chi_{2 \mathrm{~s}}\right)$ neighbour lattice sites

$$
\chi_{1 \mathrm{~s}}=\left\langle\mathbf{S}_{i} \mathbf{S}_{i+a_{1}}\right\rangle, \quad \chi_{2 \mathrm{~s}}=\left\langle\mathbf{S}_{i} \mathbf{S}_{i+a_{2}}\right\rangle,
$$

where $a_{1}=\left( \pm a_{x}, \pm a_{y}\right)$ is the nearest and $a_{2}= \pm\left(a_{x} \pm a_{y}\right)$ - the next-nearest neighbour lattice sites. In the gap equation (4.5) we omitted the $\mathbf{k}$-independent part caused by the kinematical interaction (the first term in equation (3.12)) since it gives no contribution to $d$-wave pairing [6].

To calculate the self-energy operator $\hat{\Sigma}(k, \omega)$ we employ a noncrossing approximation (or the self-consistent Born approximation) for the irreducible part of the many-particle Green functions in (3.19). In this approximation vertex corrections are neglected as in the Migdal-Eliashberg approximation and it is given by the two-time decoupling for the correlation functions in (3.19) as, e.g., given below:

$$
\left\langle X_{j^{\prime}}^{\sigma^{\prime} 0} B_{j \sigma \sigma^{\prime}}^{+} X_{i^{\prime}}^{0 \sigma^{\prime}}(t) B_{i \sigma \sigma^{\prime}}(t)\right\rangle \simeq\left\langle X_{j^{\prime}}^{\sigma^{\prime} 0} X_{i^{\prime}}^{0 \sigma^{\prime}}(t)\right\rangle\left\langle B_{j \sigma \sigma^{\prime}}^{+} B_{i \sigma \sigma^{\prime}}(t)\right\rangle .
$$


The proposed decoupling does not violate equal time correlations since in equation (4.8) $j \neq j^{\prime}$ and $i \neq i^{\prime}$. Using a spectral representation for the GF we obtain the following result for the self-energy in the noncrossing approximation:

$$
\begin{aligned}
\Sigma_{11}^{\sigma}(k, \omega) & =-\Sigma_{22}^{\bar{\sigma}}(-k,-\omega) \\
& =\frac{1}{N} \sum_{q} \iint_{-\infty}^{+\infty} \mathrm{d} z \mathrm{~d} \Omega N(\omega, z, \Omega) \lambda_{11}(q, k-q \mid \Omega) A_{11}^{\sigma}(q, z), \\
\Sigma_{12}^{\sigma}(k, \omega) & =\left(\Sigma_{21}^{\sigma}(k, \omega)\right)^{*} \\
& =-\frac{1}{N} \sum_{q} \int_{-\infty}^{+\infty} \mathrm{d} z \mathrm{~d} \Omega N(\omega, z, \Omega) \lambda_{12}(q, k-q \mid \Omega) A_{12}^{\sigma}(q, z),
\end{aligned}
$$

where

$$
N(\omega, z, \Omega)=\frac{1}{2} \frac{\tanh (z / 2 T)+\operatorname{coth}(\Omega / 2 T)}{\omega-z-\Omega} .
$$

Here we introduce the spectral density:

$$
\begin{aligned}
& A_{11}^{\sigma}(q, z)=-\frac{1}{Q_{\sigma} \pi} \operatorname{Im}\left\langle\left\langle X_{q}^{0 \sigma} \mid X_{q}^{\sigma 0}\right\rangle\right\rangle_{z+\mathrm{i} \delta}=A_{22}^{\bar{\sigma}}(q,-z), \\
& A_{12}^{\sigma}(q, z)=-\frac{1}{Q_{\sigma} \pi} \operatorname{Im}\left\langle\left\langle X_{q}^{0 \sigma} \mid X_{-q}^{0 \bar{\sigma}}\right\rangle\right\rangle_{z+\mathrm{i} \delta}=A_{21}^{\sigma}(q, z),
\end{aligned}
$$

and the electron - electron interaction functions caused by spin-charge fluctuations

$$
\begin{aligned}
& \lambda_{11}(q, k-q \mid \Omega)=g^{2}(q, k-q) D^{+}(k-q, \Omega), \\
& \lambda_{12}(q, k-q \mid \Omega)=g^{2}(q, k-q) D^{-}(k-q, \Omega),
\end{aligned}
$$

where $g(q, k-q)=t(q)-J(k-q)$ and the spectral density for the spin-charge fluctuations is defined by the commutator Green functions

$$
D^{ \pm}(q, \Omega)=-\frac{1}{\pi} \operatorname{Im}\left\{\frac{1}{4}\left\langle\left\langle n_{q} \mid n_{q}^{+}\right\rangle\right\rangle_{\Omega+\mathrm{i} \delta} \pm\left\langle\left\langle\mathbf{S}_{q} \mid \mathbf{S}_{-q}\right\rangle\right\rangle_{\Omega+\mathrm{i} \delta}\right\} .
$$

The solution of the Dyson equation ( 3.16) can be written in the Eliashberg notations as

$$
\hat{G}^{\sigma}(k, \omega)=Q_{\sigma} \tilde{G}^{\sigma}(k, \omega)=Q_{\sigma} \frac{\omega Z_{k}^{\sigma}(\omega) \hat{\tau}_{0}+\left(E_{k}^{\sigma}+\xi_{k}^{\sigma}(\omega)-\tilde{\mu}\right) \hat{\tau}_{3}+\Phi_{k}^{\sigma}(\omega) \hat{\tau}_{1}}{\left(\omega Z_{k}^{\sigma}(\omega)\right)^{2}-\left(E_{k}^{\sigma}+\xi_{k}^{\sigma}(\omega)-\tilde{\mu}\right)^{2}-\left|\Phi_{k}^{\sigma}(\omega)\right|^{2}}
$$

where

$$
\begin{aligned}
\omega\left(1-Z_{k}^{\sigma}(\omega)\right) & =\frac{1}{2}\left[\Sigma_{11}^{\sigma}(k, \omega)+\Sigma_{22}^{\sigma}(k, \omega)\right] \\
\left.\xi_{k}^{\sigma}(\omega)\right) & =\frac{1}{2}\left[\Sigma_{11}^{\sigma}(k, \omega)-\Sigma_{22}^{\sigma}(k, \omega)\right] \\
\Phi_{k}^{\sigma}(\omega) & =\Delta_{k}^{\sigma}+\Sigma_{12}^{\sigma}(k, \omega) .
\end{aligned}
$$


For the numerical solution of the system of equations (4.9)-(4.18) it is useful to introduce an imaginary frequency representation for the Green function (4.17) with $\omega=\mathrm{i} \omega_{n}=\mathrm{i} \pi T(2 n+1)$ and the spin-charge Green functions (4.16) with $\Omega=\mathrm{i} \omega_{n}=\mathrm{i} \pi T 2 n$ where $n=0, \pm 1, \pm 2, \ldots$. By using the representation for the function (4.11)

$$
N\left(\mathrm{i} \omega_{n}, z, \Omega\right)=-T \sum_{m} \frac{1}{\mathrm{i} \omega_{m}-z} \quad \frac{1}{\mathrm{i}\left(\omega_{n}-\omega_{m}\right)-\Omega}
$$

after integration in equations (4.9), (4.10) we get:

$$
\begin{aligned}
\Sigma_{11}^{\sigma}\left(k, \mathrm{i} \omega_{n}\right) & =-\frac{T}{N} \sum_{q} \sum_{m} \tilde{G}_{11}^{\sigma}\left(q, \mathrm{i} \omega_{m}\right) \lambda_{11}\left(q, k-q \mid \mathrm{i} \omega_{n}-\mathrm{i} \omega_{m}\right), \\
\Sigma_{12}^{\sigma}\left(k, \mathrm{i} \omega_{n}\right) & =\frac{T}{N} \sum_{q} \sum_{m} \tilde{G}_{12}^{\sigma}\left(q, \mathrm{i} \omega_{m}\right) \lambda_{12}\left(q, k-q \mid \mathrm{i} \omega_{n}-\mathrm{i} \omega_{m}\right) .
\end{aligned}
$$

The interaction functions are given by

$$
\begin{aligned}
& \lambda_{11}\left(q, k-q \mid \mathrm{i} \omega_{\nu}\right)=g^{2}(q, k-q) D^{+}\left(q, \mathrm{i} \omega_{\nu}\right), \\
& \lambda_{12}\left(q, k-q \mid \mathrm{i} \omega_{\nu}\right)=g^{2}(q, k-q) D^{-}\left(q, \mathrm{i} \omega_{\nu}\right) .
\end{aligned}
$$

Here we have $G_{11}^{\sigma}\left(k, \mathrm{i} \omega_{m}\right)=-G_{22}^{\bar{\sigma}}\left(k,-\mathrm{i} \omega_{m}\right), G_{12}^{\sigma}\left(k, \mathrm{i} \omega_{m}\right)=G_{21}^{\sigma}\left(k,-\mathrm{i} \omega_{m}\right)$.

A linearized system of the Eliashberg equations (4.18) for $T \leqslant T_{\mathrm{c}}$ has the following form:

$$
\begin{aligned}
\tilde{G}_{11}^{\sigma}\left(k, \mathrm{i} \omega_{n}\right)= & \frac{1}{\mathrm{i} \omega_{n}-E_{k}+\tilde{\mu}-\Sigma_{11}^{\sigma}\left(k, \mathrm{i} \omega_{n}\right)} \\
\Phi^{\sigma}\left(k, \mathrm{i} \omega_{n}\right)= & \Delta_{k}^{\sigma}+\phi^{\sigma}\left(k, \mathrm{i} \omega_{n}\right)=\frac{T}{N} \sum_{q} \sum_{m}\{2 J(k-q) \\
& \left.-\lambda_{12}\left(q, k-q \mid \mathrm{i} \omega_{n}-\mathrm{i} \omega_{m}\right)\right\} \tilde{G}_{11}^{\sigma}\left(q, \mathrm{i} \omega_{m}\right) \tilde{G}_{11}^{\bar{\sigma}}\left(q,-\mathrm{i} \omega_{m}\right) \Phi^{\sigma}\left(q, \mathrm{i} \omega_{m}\right) .
\end{aligned}
$$

At first the system of equations for the normal GF (4.24), (4.20) should be solved for a given concentration of electrons

$$
\frac{n}{1-n / 2}=1+\frac{2 T}{N} \sum_{k} \sum_{n=-\infty}^{\infty} \tilde{G}_{11}\left(k, \mathrm{i} \omega_{n}\right) .
$$

Then, the eigenvalues and eigenfunctions of the linear equation for the gap function (4.26) should be calculated to obtain the superconducting transition temperature $T_{\mathrm{c}}$ and the $(q, \omega)$ dependence gap function.

For numerical calculations one has to introduce a model for the charge-spinfluctuation functions in (4.16). By taking into account only the spin-fluctuation contribution, we can write them in the form:

$$
D_{\mathrm{s}}^{-}\left(q, \mathrm{i} \omega_{\nu}\right)=-D_{\mathrm{s}}^{+}\left(q, \mathrm{i} \omega_{\nu}\right)=\chi_{\mathrm{s}}(q) \int_{0}^{+\infty} \frac{2 z \mathrm{~d} z}{z^{2}+\omega_{\nu}^{2}} \chi_{\mathrm{s}}^{\prime \prime}(z)
$$


where we have introduced for the spin-fluctuation susceptibility a model representation (see, e.g., $[11,18]$ )

$$
\begin{aligned}
\chi_{\mathrm{s}}^{\prime \prime}(q, \omega) & =-\frac{1}{\pi} \operatorname{Im}\left\langle\left\langle\mathbf{S}_{q} \mid \mathbf{S}_{-q}\right\rangle\right\rangle_{\omega+\mathrm{i} \delta} \\
& =\chi_{\mathrm{s}}(q) \chi_{\mathrm{s}}^{\prime \prime}(\omega)=\frac{\chi_{0}}{1+\xi^{2}\left(\mathbf{q}-\mathbf{Q}_{\mathrm{AF}}\right)^{2}} \tanh \frac{\omega}{2 T} \frac{1}{1+\left(\omega / \omega_{\mathrm{s}}\right)^{2}}
\end{aligned}
$$

with the characteristic AFM correlation length $\xi$ and the spin-fluctuation energy $\omega_{\mathrm{s}} \simeq J$. To fix the constant $\chi_{0}$ in (4.28) we can use for the spin-fluctuation susceptibility the following normalizing condition:

$$
\frac{1}{N} \sum_{i}\left\langle\mathbf{S}_{i} \mathbf{S}_{i}\right\rangle=\frac{1}{N} \sum_{q} \chi_{\mathrm{s}}(q) \int_{-\infty}^{+\infty} \frac{\mathrm{d} z}{\exp (z / T)-1} \chi_{\mathrm{s}}^{\prime \prime}(z)=\frac{3}{4} n .
$$

For the low temperature, $T \ll \omega_{\mathrm{s}}$, we can integrate over frequency in equation (4.29):

$$
\int_{-\infty}^{+\infty} \frac{\mathrm{d} z}{\exp (z / T)-1} \chi_{\mathrm{s}}^{\prime \prime}(z) \simeq \int_{0}^{+\infty} \mathrm{d} z \chi_{\mathrm{s}}^{\prime \prime}(z) \simeq \int_{0}^{+\infty} \frac{\mathrm{d} z}{1+\left(z / \omega_{\mathrm{s}}\right)^{2}}=\frac{\pi}{2} \omega_{\mathrm{s}} .
$$

Therefore, for the constant $\chi_{0}$ we get from (4.29)

$$
\chi_{0}=\frac{3 n}{2 \pi \omega_{\mathrm{s}} C_{1}}, \quad C_{1}=\frac{1}{N} \sum_{q} \frac{1}{1+\xi^{2} q^{2}} \simeq \frac{\pi}{\xi^{2}} \ln \left(1+\xi^{2} / \pi\right) .
$$

Here $\xi$ and $q$ are dimensionless quantities ( $\xi / a$ and $q a$ where $a$ is the lattice constant). In the approximation (4.27), we get for the interaction functions (4.22), (4.23):

$$
-\lambda_{11}\left(q, k-q \mid \mathrm{i} \omega_{\nu}\right)=\lambda_{12}\left(q, k-q \mid \mathrm{i} \omega_{\nu}\right)=g^{2}(q, k-q) \chi_{\mathrm{s}}(k-q) F_{\mathrm{s}}\left(\mathrm{i} \omega_{\nu}\right),
$$

where

$$
F_{\mathrm{s}}\left(\mathrm{i} \omega_{\nu}\right)=\int_{0}^{+\infty} \frac{2 z \mathrm{~d} z}{z^{2}+\omega_{\nu}^{2}} \frac{1}{1+\left(z / \omega_{\mathrm{s}}\right)^{2}} \tanh \frac{z}{2 T} .
$$

By using the model (4.28), we get for the static spin correlation functions (4.7):

$$
\begin{aligned}
& \chi_{1 \mathrm{~s}}=\left\langle\mathbf{S}_{i} \mathbf{S}_{i+a_{1}}\right\rangle=\frac{1}{N} \sum_{q} \gamma(q)\left\langle\mathbf{S}_{q} \mathbf{S}_{-q}\right\rangle, \\
& \chi_{2 \mathrm{~s}}=\left\langle\mathbf{S}_{i} \mathbf{S}_{i+a_{2}}\right\rangle=\frac{1}{N} \sum_{q} \gamma^{\prime}(q)\left\langle\mathbf{S}_{q} \mathbf{S}_{-q}\right\rangle,
\end{aligned}
$$

where

$$
\left\langle\mathbf{S}_{q} \mathbf{S}_{-q}\right\rangle=\chi_{\mathrm{s}}(q) \int_{-\infty}^{+\infty} \frac{\mathrm{d} z \chi_{\mathrm{s}}^{\prime \prime}(z)}{\exp (z / T)-1} \simeq \chi_{\mathrm{s}}(q) \frac{\pi}{2} \omega_{\mathrm{s}} \simeq \frac{3 n}{4 C_{1}} \frac{1}{1+\xi^{2}(\mathbf{q}-\mathbf{Q})_{\mathrm{AF}}^{2}} .
$$

Therefore, we have obtained a closed system of equations which should be solved numerically as it was recently done for the spin-polaron model in [8]. 


\section{Conclusions}

In the present paper the theory of the quasiparticle spectrum and superconducting pairing in the $t-t^{\prime}-J$ model (2.2) in a paramagnetic state is formulated. By employing the equation of motion method for the two-time GF [2] and differentiating it over two times, $t$ and $t^{\prime}$, we easily obtained a self-consistent system of equations for the matrix GF (4.17) and self-energy (4.9), (4.10) in the noncrossing approximation, equation (4.8). The latter is equivalent to the MigdalEliashberg approximation which neglects vertex corrections. Though in the $t-t^{\prime}-J$ model (2.2) we have no small parameter as in the electron-phonon model considered by Eliashberg [3], the vertex renormalization in the former may be not so important as it was proved for the spin-polaron representation of the $t-t^{\prime}-J$ model (2.2) (see, e.g., [19]). All the calculations are performed in the real time representation, though the imaginary frequency representation which is more convenient for a numerical study is given for the linearized system of Eliashberg equations (4.24), (4.26).

However, the theory is not fully self-consistent in that respect that a phenomenological model for effective electron-electron coupling due to spin fluctuations is to be proposed, equation (4.27), to enable a numerical study of temperature and doping dependence of the quasiparticle spectrum and superconducting pairing in the model (2.2). The results of numerical calculations and the comparison with other approaches will be presented in a separate paper.

\section{Acknowledgements}

Partial financial support by the INTAS-RFBR Program, Grant No 95-591, is acknowledged.

\section{References}

1. Zubarev D.N. On the theory of superconductivity. // Doklady AN SSSR, 1960, vol. 132, No. 5, p. 1055-1058 (in Russian).

2. Zubarev D.N. Two-time Green functions in the statistical physics. // Uspekhi Fiz. Nauk, 1960, vol. 71, No. 1, p. 71-116 (in Russian).

3. Eliashberg G.M. Interaction of electrons with lattice vibrations in a superconductor. // J. Exp. Teor. Fiz., 1960, vol. 38, No. 3, p. 966-976 (in Russian); Temperature Green's function for electrons in a superconductor. // ibid., 1960, vol. 39, No. 5, p. 1437-1441 (in Russian).

4. Vujičić G.M., Petru Z.K., Plakida N.M. On the equations of superconductivity for electron-ion model of metals. // Teor. Mat. Fiz., 1981, vol. 46, No. 1, p. 91-98 (in Russian).

5. Zaitsev R.O., Ivanov V.A. On the possibility of pair condensation in the Hubbard model. // Fiz. Tverd. Tela, 1987, vol. 29, No. 8, p. 2554-2556 (in Russian); Superconductivity in the Hubbard model. // ibid., No. 10, p. 3111-3119 (in Russian). 
6. Plakida N.M., Yushankhai V.Yu., Stasyuk I.V. On the role of kinematical and exchange interactions in superconducting pairing of electrons in the Hubbard model. // Physica C, 1989, vol. 160, p. 80-88.

7. Yushankhai V.Yu., Plakida N.M., Kalinay P. Superconducting pairing in the meanfield approximation for the $t-J$ model: Numerical analysis. // Physica C, 1991, vol. 174, p. 401-406.

8. Plakida N.M., Oudovenko V.S., Horsch P., Liechtenstein A.I. Superconducting pairing of spin polarons in the $t-J$ model. // Phys. Rev. B, 1997, vol. 55, No. 18, p. R11997R12000.

9. Izyumov Yu.A., Letfulov B.M. Spin fluctuations and superconductivity in the Hubbard model with a strong Coulomb repulsion. // J. Phys.: Cond. Matt., 1991, vol. 3, p. 53735391.

10. Plakida N.M., Hayn R. Superconducting pairing in the singlet band of the Emery model. // Zeit. Physik B, 1994, vol. 93, p. 313-319.

11. Prelovśek P. Electron Green's function in the planar $t-J$ model. // Zeit. Physik B, 1997, vol. 103, p. 363-368.

12. Plakida N.M. High-temperature Superconductivity. Heidelberg, Springer, 1995.

13. Plakida N.M. Spin fluctuation superconducting pairing in copper oxides. // Philosophical Magazine B, 1997, vol. 76, No. 5, p. 771-795.

14. Izyumov Yu.A. The $t-J$ model for strongly correlated electrons and high- $T_{\mathrm{c}}$ superconductors. // Uspekhi Fiz. Nauk, 1997, vol. 167, No. 5, p. 465-497 (in Russian).

15. Anderson P.W. The resonating valence bond state in $\mathrm{La}_{2} \mathrm{CuO}_{4}$ and superconductivity. // Science, 1987, vol. 235, p. 1196-1198.

16. Emery V. Theory of high- $\mathrm{T}_{\mathrm{c}}$ superconductivity in oxides. // Phys. Rev. Lett., 1987, vol. 58, No. 26, p. 2794-2797.

17. Zhang F.C., Rice T.M. Effective Hamiltonian for the superconductiviting Cu oxides. // Phys. Rev. B, 1988, vol. 37, No. 7, p. 3759-3761.

18. Jaklič J., Prelovśek P. Anomalous spin dynamics in doped quantum antiferromagnets. // Phys. Rev. Lett., 1995, vol. 74, No. 17, p. 3411-3414; Universal charge and spin dynamics in optimally doped antiferromagnets. // Phys. Rev. Lett., 1995, vol. 75, No. 17 , p. $1340-1343$.

19. Liu Z., Manousakis E. Dynamical properties of a hole in a Heisenberg antiferromagnet. // Phys. Rev. B, 1992, vol. 45, No. 5, p. 2425-2437. 


\section{Підхід функцій Ґріна в теорії надпровідності для} $t-J$ моделі

\section{М.М.Плакіда}

Об’єднаний інститут ядерних досліджень, 141980 Дубна, Росія

Отримано 28 січня 1998 р.

Розвинуто теорію надпровідного спарювання, що відбувається завдяки обмінним та кінематичним взаємодіям у $\boldsymbol{t}-\boldsymbol{J}$ моделі в парамагнітному стані. Отримано рівняння Дайсона для матриці функцій Ґріна через оператори Хабарда у неперехресному наближенні. Запропоновано лінеаризовану самоузгоджену систему рівнянь Еліашберга для вивчення температурної та концентраційної залежності квазічастинкового спектру дірок у нормальному стані, а також для знаходження температури надпровідного фазового переходу і симетрії щілини.

Ключові слова: надпровідність, квазічастинка, рівняння Дайсона, функції Ґріна, оператори Хабарда

PACS: $74.20 .-z, 74.20 . M n, 74.72 .-h$ 
